merely a review of the current status of clinical and experimental trials.

The first chapter reviews lymphocyte and antibody responses to experimental tumours. Although antibody-lymphocyte interactions are covered, the currently fashionable natural killer cell is surprisingly scantily described in only one short paragraph. The account in the second chapter, of the possibility of tumour localization of antibody directed against tumour or tumour stroma, follows a historical line, and whether there really is potential for this approach clinically is unclear. The use of cytotoxic drugs coupled to antibody, and antibody-drug interactions is briefly mentioned, but not reviewed elsewhere in this volume. The third chapter extensively reviews the current concept of the immune macrophage, and macrophages activated by immunotherapeutic agents such as BCG and C. parvum and their proposed mechanisms of tumour-cell destruction. Further chapters (5 and 6 ) review the evidence that such immune potentiators and other agents, including levamisole, double-stranded RNA and endotoxin, exert their effects through changes in cyclic GMP and AMP levels in macrophages and lymphocytes, and discusses the potential of such agents for future therapeutic application. The history and wide immunological effects of levamisole are further covered in Chapter 7, together with its clinical application to a wide variety of malignant diseases. The realistic overall conclusion from these trials, stressed by the authors, is that they have not yet yielded conclusive evidence of benefit, but that further trials are warranted. The clinical application of levamisole to breast cancer is covered in greater depth in Chapter 8 where the detailed results of clinical trials are presented, and the interpretation is more optimistic. Chapters 9 and 10 cover the immunotherapy of experimental and human cancer with RNA extracts from syngeneic and xenogeneic tumourimmune lymphoid cells, particularly the application to melanoma, where again, although the results are suggestive of benefit, further trials are needed to resolve the problem. The effect of neuraminidase on cellular immunity is covered in another chapter (17), but greater emphasis is put on the general effects of immune responses in vivo and in vitro rather than only the effect directly on tumour-cell surfaces and in vivo behaviour of enzyme-treated cells. Only a few chapters of this book are devoted to treatment of specific malignancies, and here too the background immunology is stressed, rather than a review of passed trials. With melanoma, evidence for cellular and humoral responses is covered, before a brief outline of immunotherapy trials covering BCG, immune RNA, levamisole, etc. (Chapter 11). The whole of Chapter 12 is devoted to the immunobiology of intracranial tumours, before their attempted immunotherapy is described in Chapter 13. The earlier Chapter 4 similarly outlines the evidence for the immunogenicity of human breast cancer, based mainly on the MTV animal model, before describing briefly some clinical trials. Three chapters (14-16) are devoted to the possible non-specific adoptive immunotherapy of cell leukaemia, basing clinical potential on the results of work with the AKR mouse leukaemia.

Clearly future immunotherapy must be rational rather than empirical. This book reviews some of these rational approaches for future development and indicates to the reader where immunotherapy might be going, rather than simply where it has been.

\section{V. Pimм}

\section{Histological Typing of Upper Respiratory Tract Tumours (International Histo- logical Classification of Tumours, No.}

19). By K. Shanmugaratnam (1978).

Geneva: W.H.O. 56 pp. Sw.fr.285.

This volume in the WHO International Histological Classification of Tumours covers tumours of the ear, nose, nasopharynx and larynx. It is likely to prove one of the most valuable volumes in the series. Within the anatomical regions surveyed, one may encounter tumours of the most varied histogenesis, in addition to tumours of the respiratory epithelia. Tumours of salivary, odontogenic, bony, neural and lymphoreticular origin may all present as "ENT" problems, and the pathologist who has to reach a diagnosis on the often unsatisfactory specimens provided by the ENT surgeon needs to be familiar with all these tumours, and more besides. He will find this volume an invaluable guide. The definitions are crisp and dogmatic, and the colour photographs are of excellent quality and show the essential features of each lesion. The section on tumour-like lesions is particularly valuable, as these often 
cause diagnostic problems in this area. Strongly recommended.

O. G. Dodge

The Wayward Cell-Cancer-Its Origins, Nature and Treatment. By $V$. Richardos (1978). Los Angeles: University of California Press. 407 pp. US\$12.50,

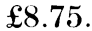

This is described as "an updated version" of the successful first edition published in 1972. The updating is apparently not done chapter by chapter but by adding a final chapter on "New Methods of Cancer Treatment-1972-1977". The book is avowedly directed to "any intelligent person willing to make a modest effort" but, like so many books of this kind, the intelligent layman would find it tough reading from time to time.

Part I deals with the biology of cancer, and discusses in some detail the structure and function of living cells; growth, differentiation and genetics; the transfer of inheritance; viruses, immunity and the synthesis of life; normal and cancer cells and viral carcinogenesis. The intelligent layman would require more than a "modest effort" to digest and retain an understanding of the molecular biology discussed throughout this section of the book.

Part II describes the clinical aspects of cancer, and Part III the various approaches to treatment-surgery, radiotherapy, chemotherapy and immunotherapy. Having displayed his own considerable erudition over a vast field it is perhaps surprising that the author should need to invite Denise Scott to deal, in Part IV, with the psycho-social problems of cancer-smoking and cancer, psychological problems, quackery, and death from cancer. These important subjects are, however, well done.

The final section dealing with developments between 1972 and 1977 is somewhat repetitious, but is virtually a cohesive monograph on its own, well documented and of interest to clinical oncologists of all disciplines.

This has been a good book of its kind since
1972 and the updated version will still find a place in selected libraries.

E. EASSON HLA and H-2. Basic Immunogenetics,
Biology and Clinical Relevance. Current Topics in Immunology Vol. 9. By $\mathrm{H}$. Festenstein and P. Demant (1978). Berks: Edward Arnold. 212 pp. $£ 9.00$.

All mammalian species so far studied, and perhaps all vertebrate species, have an area of genetic material which is of primary importance in determining the fate of tissue grafts.

The study of these major histocompatibility systems has proved to be one of the most fertile areas of current biological research, and has paid handsome dividends in both fundamental immunology and clinical medicine.

The rapid advance of immunogenetics has created a gulf between specialized publication and superficial review, which the authors bridge very successfully with a book that provides a thorough, though entirely readable, account of the HLA and H-2 systems. The first chapter introduces transplantation-related immunology and lays the foundations for the subsequent chapters. These deal with the HLA system and its relevance to transplantation, the H-2 system of the mouse, the biology of the MHS, including a discussion of the H-2-linked immune-response genes, and the association between HLA and certain clinical disorders.

The scope of the book is broad and the authors do not limit themselves to purely theoretical aspects of their subject. For instance, as well as considering the relevance of HLA specificities to renal transplantation, the methodologies and interpretative difficulties of tissue matching and the logistic considerations of tissue typing are also described.

The standard of presentation throughout is excellent and the book can be warmly recommended to clinicians, research workers in related fields and students alike.

M. Moore

I. KIMBER 